\title{
Una nueva cultura del trabajo para un mercado laboral y unas sociedades inclusivas
}

\author{
$M^{\mathrm{a}}$ Luz de la Cal
}

Facultad de Relaciones Laborales y Trabajo Social, Universidad del País Vasco / Euskal Herriko Unibertsitatea (UPV/EHU)

<luz.cal@ehu.es>

\section{Madeleine Huber}

Estudiante del máster de Economía Social y Solidaria, Universidad del País Vasco / Euskal Herriko Unibertsitatea (UPV/EHU)

$<$ madeleine.huber92@gmx.de>

\begin{abstract}
Azken hamarkadetako gizarteratze-politikak nabarmendu izan dira kalteberatasun eta gizartebazterketa egoeran dauden biztanleen ikuspegian zentratu izanagatik. Horren asmoa izan da kasu bakoitzean agertutako trabak aintzat hartuta inklusiorako ibilbideak indibidualizatutako moduen eskuartzeen bidez diseinatzea. Ikuspegi horien baliagarritasunaz beste, aldi berean, zentzuzkoa ematen du gizarteratzeko guneak zabaldu ahal izateko aldaketa batzuk sartu ahal izatea egungo gizarte garaikideetan. Artikulu honen xedea da langabezia eta enpleguaren ezegonkortasunaren inguruan azken urteotako aukera horietako batzuk azaldu eta berauen inguruko hausnarketa burutzea. Horiek guztiek proposatzen dute lanaren kultura berri baten alde egitea, modu horretan biztanle gehiagok sarbidea izateko bai enplegu bai eskubide sozialen eremuan.
\end{abstract}

\section{GAKO-HITZAK:}

Langabezia, flexisegurtasuna, itzulerako eskubide sozialak, trantsiziozko lan-merkatuak, enpresetako boluntariotza, ekonomia purpura.
En las últimas décadas, las políticas de inclusión social han venido marcadas por una perspectiva muy centrada en las personas en situación de vulnerabilidad o de exclusión. Se trataba de individualizar las intervenciones con base en la idea de que los itinerarios de inclusión deben diseñarse atendiendo a cuáles son las barreras en cada caso. Sin dejar de considerar la validez de estos enfoques, parece pertinente plantear, al mismo tiempo, algunos cambios en nuestras sociedades que favorezcan una ampliación de los espacios de inclusión. El objetivo de este artículo es exponer y reflexionar en torno a algunas de las alternativas que, a lo largo de los últimos años, se han ido planteando como respuesta al desempleo y a la precarización de una buena parte del empleo. Todas ellas proponen una nueva cultura del trabajo que permita que un mayor número de personas accedan al empleo y a los derechos sociales.

\section{Palabras Clave:}

Desempleo, flexiguridad, derechos sociales de giro, mercados transicionales de trabajo, voluntariado empresarial, economía púrpura. 


\section{La rigidez del modelo de inserción sociolaboral}

La anterior crisis que sufrió el capitalismo a mediados de los setenta ya puso de manifiesto la importante escasez de empleos derivada de sistemas cada vez más productivos y también de cambios sociales que agregaban nuevos sectores de población al mercado laboral, como el de las mujeres, que poco a poco fueron abandonando su dedicación exclusiva a las tareas del hogar y los cuidados.

En gran medida, las políticas económicas implementadas han abordado el problema del desempleo como un desequilibrio entre oferta y demanda de trabajo que se corrige en las épocas de expansión económica o mediante el ajuste salarial. Se trata de políticas para devolver a las economías a la senda del crecimiento y la creación de empleo, destruyendo sobreacumulaciones de capital, relanzando sectores a través de las nuevas tecnologías y abriendo mercados, y también a través de recortes del gasto público, liberalizaciones de algunos sectores, y de una importante flexibilización y desregulación laboral, lo que ha tenido importantes costes en términos sociales. Si bien ha habido épocas en las que el desempleo se ha reducido de manera considerable, en algunos colectivos y regiones nunca se ha llegado a eliminar. Para explicar estos casos, se ha aludido a desajustes de tipo estructural en cuanto a la formación de la mano de obra y los requerimientos del sector productivo, y también a las rigideces institucionales del mercado laboral.

En general, no se han cuestionado las bases del modelo sociolaboral, de manera que el empleo a tiempo completo y una trayectoria laboral sin interrupciones han seguido siendo el pasaporte al pleno disfrute de derechos sociales y laborales. Ello a pesar de que cada vez sectores más amplios de la población activa quedaban al margen de este modelo, por la temporalidad o la parcialidad de sus empleos, o porque conocían episodios de paro en sus historias laborales. Las tasas de pobreza, incluso entre la población trabajadora, son muestra de que la expansión no ha alcanzado a todos los grupos de población. Ello no ha impedido que el sistema haya conocido un periodo significativo de crecimiento económico ni que las tasas de beneficio se hayan recuperado.

La crisis que se inició en 2008 dejó al descubierto la debilidad de las bases de la expansión económica anterior, sobre todo en el sur de Europa. En los primeros años, sí que hubo algunos pronunciamientos sobre la 'necesidad de refundar el capitalismo'. Incluso la primera respuesta europea a la crisis financiera confió en las recetas keynesianas, favoreciendo la expansión del gasto público para estabilizar la economía. Sin embargo, el rescate de los bancos por parte de los Estados, con el consiguiente incremento del déficit público, pronto precipitó a algunos de estos Estados a los denominados 'rescates' y a procesos de consolidación fiscal. Las políticas de austeridad han sido de obligado cumplimiento para estos Estados, pero en otros no rescatados se siguió este mismo ejemplo. Ello sería una muestra de que tan sólo estamos asistiendo a un episodio más de la respuesta neoliberal a las crisis que nos acompaña desde los años ochenta, o como algunos trabajos la denominan, la versión "austeritaria" de las políticas deflacionistas (Gálvez y Rodríguez, 2013b: 120).

En todos estos años de políticas de ajuste, de austeridad y control del gasto público, de liberalización y privatización de sectores, de flexibilización y desregulación laboral, no ha llegado a consolidarse un modelo, un nuevo régimen de acumulación que dinamice la economía capitalista y que estabilice la creación de empleo. Ya no se puede confiar en que el modelo siga funcionando, en el sentido de que se llegue a un equilibrio, inestable pero aceptable, entre las dimensiones económicas, sociales, políticas y culturales, de forma que se pueda reducir al mínimo la exclusión (si es que hay algún nivel mínimo aceptable) dentro del sistema capitalista.

Seguir confiando en el trabajo asalariado y remunerado como el gran factor de integración social no es la respuesta ante los problemas de exclusión de un número cada vez más importante de personas. Por una parte, porque el trabajo es cada vez menos necesario para la acumulación de capital. La industria genera cada vez menos puestos de trabajo debido a la automatización, y los que genera están crecientemente amenazados por la deslocalización a países con costes laborales más bajos. En el sector servicios, también van disminuyendo los puestos de trabajo más rutinarios con la introducción de las tecnologías de la información y la comunicación. Por otro lado, porque una parte importante de los puestos de trabajo que se generan son incompatibles con la reproducción social, por su precariedad.

Durante los años en los que ilusoriamente creímos haber superado la anterior crisis, el crecimiento económico no consiguió reducir las tasas de pobreza de manera sustancial. La vulnerabilidad y fragilidad en la esfera económico-laboral han provocado también problemas de tipo relacional y en otras áreas de la integración, como la vivienda o la salud $^{1}$. Se trataba de un crecimiento profundamente desequilibrado en cuanto al reparto de sus frutos, tal y como ilustran todos los indicadores de desigualdad en la distribución de la renta y la riqueza. En los países del sur de Europa, no se aprovecharon estos años para desarrollar plenamente los Estados del bienestar, dado que se priorizó la rebaja de la presión fiscal, sobre todo para los grandes negocios y los sectores de renta alta.

${ }^{1}$ Algunas investigaciones (Castel, 1997; Paugam, 1996, 2000) han dado cuenta de los vínculos entre una débil inserción laboral y la fragilidad en otras esferas de la integración. 
A la crisis económica y laboral, se le ha ido sumando también la de los cuidados, agudizada por el envejecimiento poblacional que sufren una buena parte de los países más avanzados del mundo. La crisis medioambiental es otro de los flancos incontrolados del modelo y que no se está abordando de manera decidida, a pesar de las numerosas evidencias sobre el cambio climático.

La gran recesión que se inició en 2008 no hizo sino acentuar los problemas descritos y extenderlos a más capas de la población. Lo que era precariedad e inestabilidad ha derivado en una auténtica crisis social, dado que los mecanismos de protección social no han respondido de manera adecuada. Hay nuevas situaciones de necesidad no cubiertas por los sistemas convencionales de protección social. En parte, se les ha dado respuesta mediante el desarrollo de prestaciones asistenciales, como las de garantía de rentas mínimas, lo que supone el abandono de un enfoque basado en derechos.

Incluso aunque pueda haber periodos puntuales de crecimiento en los que las tasas de desempleo se reduzcan, no parece factible que con el actual modelo de organización sociolaboral podamos conseguir la plena integración laboral de todas las personas en edad de trabajar. Parece razonable plantearse la necesidad de cambiar el citado modelo, dado que las circunstancias en las que fue diseñado y en que se convirtió en la norma han variado de manera sustancial. La jornada de 40 horas semanales se instauró entre los años setenta y ochenta en numerosos países, es decir, que han transcurrido unos 45 años. En este tiempo, los procesos productivos se han transformado de manera que hace falta mucha menos mano de obra para llevarlos a cabo, y también ha habido transformaciones sociales notabilísimas.

Seguramente, consideraríamos un desatino intentar hoy día producir bienes y servicios con tecnologías de hace 45 años, o gestionar los negocios y financiarlos con métodos de aquella época, por mencionar algunos procesos en los que ha habido cambios importantísimos. Sin embargo, y a pesar de las numerosas modificaciones que se han llevado a cabo en las legislaciones laborales y de seguridad social, el núcleo de la organización de los tiempos de trabajo y de la adquisición de los derechos sociales permanece prácticamente invariable. La jornada de referencia sigue siendo de 40 horas semanales en muchísimos casos, y los tiempos de cotización necesarios para acceder a prestaciones contributivas apenas han variado. Todo ello a pesar de los incrementos de productividad, de unas vidas laborales más cortas, por la tardía incorporación al empleo, del incremento en la esperanza de vida y de cambios sociales que afectan a la oferta de trabajo disponible en nuestras sociedades, como la salida de las mujeres del espacio doméstico y su incorporación (en muchos casos, parcial) al mercado laboral.

Las situaciones que se salen de esta norma son cada vez más frecuentes, pero los riesgos que llevan asociados no están contemplados, así que originan vulnerabilidad, precariedad o incluso exclusión social. Se prioriza lo urgente, el enfoque asistencial, pero no se atacan los factores estructurales que originan estas situaciones. Este modelo de organización sociolaboral que apenas se ha adaptado a las nuevas circunstancias productivas y sociales es como una especie de cepo que nos tiene atrapados. A algunos/as, en empleos precarios y con poca cobertura social para el futuro; a otros/as, en empleos a jornada completa a veces difícilmente conciliables con la vida personal y familiar; mientras que otros sectores no encuentran lugar reconocido y legitimado en esta sociedad, porque no tienen empleo. Un símil válido podría ser el de un mismo paraguas para cobijar a un número creciente de personas ante condiciones climáticas cada vez más adversas, personas a las que no se les da opción de moverse hacia lugares donde haya cobijo.

Los conceptos que se presentan a continuación son diferentes propuestas para avanzar hacia una nueva cultura del trabajo. Todos ellos tratan de contemplar, reconocer y repartir:

- Todo el trabajo que es útil y necesario en nuestras sociedades, y que va mucho más allá del empleo (sobre todo, el trabajo doméstico y los cuidados a personas dependientes, aunque no exclusivamente).

- Actividades sociales, políticas, culturales, sindicales que nutren nuestras sociedades, y también las necesarias actividades formativas para que la mano de obra se adapte a los continuos cambios en los procesos productivos.

- Trabajo voluntario que contribuya de manera positiva a la participación económica, social y democrática, y que aumente la sensibilidad de la población ante problemas globales, como la desigualdad o los retos medioambientales.

\section{Propuestas para cimentar un nuevo modelo de inserción sociolaboral}

\subsection{Los enfoques sobre derechos sociales de giro $y$ estado profesional de las personas}

Estas propuestas surgieron a finales de los noventa como respuesta a los efectos negativos que habían creado las políticas de flexibilidad laboral implementadas en buena parte de los países europeos en los años anteriores. Estas políticas de flexibilidad, si bien pudieron haber creado oportunidades de entrada de ciertos grupos en el mercado de trabajo y un uso más flexible de la mano de obra que precisaba el sector empresarial, provocaron también un importante incremento de las formas atípicas de empleo, niveles bajos de seguridad en el ingreso y de cobertura social así como inestabilidad laboral. Pero es que, además, las citadas propuestas, señalaban que una estrategia de flexibilidad centrada exclusivamente en la regulación 
del volumen de trabajo, podría ser incompatible con otras formas de flexibilidad que garantizarían la competitividad a largo plazo, como son la capacidad de la empresa para ajustarse a una demanda variable en volumen y composición, y la aptitud de las plantillas para cambiar de puesto de trabajo y tareas en la organización.

Las nociones de 'estado profesional de las personas' y de 'derechos sociales de giro' desarrolladas por Supiot constituyen una vía para proporcionar seguridad en condiciones de inseguridad. La flexibilidad debe ser entendida como búsqueda de una respuesta colectivamente eficiente a las situaciones económicas imprevisibles. Las incertidumbres no deben reducirse a un riesgo previsible, sino que es necesario el tratamiento de la incertidumbre en toda su amplitud; debe interiorizarse, no externalizarse y tratarse $a$ posteriori. La flexibilidad implica, entonces, la necesidad de formar, mantener y desarrollar, a lo largo de su ciclo de vida, las capacidades de las personas. Para ello, las preocupaciones relativas al capital humano deben ser prioritarias -su financiación, sus características diversas y evolutivas, o las modalidades de formación y mantenimiento-, y esto sólo puede basarse en la seguridad del empleo. Para que la mano de obra sea flexible y adquiera nuevas competencias, necesita un marco de seguridad que le permita hacer previsiones a largo plazo (Supiot, 1999a).

La noción de estado profesional de las personas surge del agotamiento del modelo de empleo de los años posteriores a la Segunda Guerra Mundial. Este modelo nunca se extendió a toda la población, ya que había importantes colectivos, como el de las mujeres, que no participaban en el mercado laboral. Con todo, sí que puede decirse que la mayoría de la población que trabajaba en la esfera mercantil lo hacía como asalariada y en empleos estándar. Hoy día, dados los cambios acaecidos en los mercados laborales, este tipo de empleo no puede constituir el paradigma de la relación de trabajo, ya que representa sólo una parte de ella, aunque muy importante todavía. El número de personas excluidas de este modelo de empleo no deja de crecer: se trata de población desempleada (que puede tener derechos sociales, pero no trabajo). También de personas que están permanentemente con contratos temporales o a tiempo parcial, y de los trabajadores y trabajadoras pobres (tienen trabajo, pero sus ingresos no son suficientes para superar el umbral de pobreza). El estado profesional de las personas, al contrario que el estatus salarial, engloba los periodos de empleo en un sistema más comprensivo de derechos sociales. Este estado profesional empieza a aparecer en los sistemas de seguridad social que han sido adaptados para asegurar la continuidad de los derechos sociales ante la discontinuidad de las posiciones en el mercado de trabajo. Pero se trata de dar un paso más, y no de limitarse a la protección contra los riesgos. Se trataría de permitir a toda persona trabajadora conducir libremente su vida profesional (Supiot, 1999b).
Los derechos sociales de giro, o a cuenta, se basan no en el riesgo, sino en un crédito previamente adquirido. Se basan en decisiones voluntarias de las personas que tienen esos derechos, en vez de en un acontecimiento de riesgo. Son derechos adquiridos durante los periodos de empleo, pero también pueden adquirirse en otras situaciones de trabajo (trabajo independiente o no profesional) o del Estado, de los seguros o de organismos paritarios. Nutren el estado profesional de las personas porque permiten a los individuos beneficiarse de periodos remunerados de no trabajo, y pueden proporcionar una forma de redistribuir voluntariamente periodos de trabajo y de inactividad remunerados a lo largo del ciclo de la vida. Estos derechos llegarían a adquirirse, de forma progresiva, mediante el ejercicio de un trabajo. Permiten una liberación del tiempo y proporcionan de este modo un espacio para articular la flexibilidad del trabajo, a condición de que el trabajo generador de derechos se extienda más allá del trabajo asalariado. El individuo conseguiría así formar un crédito sobre unos fondos que podría utilizar libremente en diversos momentos de su vida (Supiot, 1999a). De esta forma, se permite a la persona sustraerse momentáneamente de las restricciones de su actividad profesional, actual para dedicarse a otra actividad socialmente útil (cuidar hijos e hijas, o padres y madres mayores, montar su propia empresa, participar en la vida sindical o asociativa, en acciones humanitarias).

El planteamiento de Supiot va mucho más allá de ofrecer un marco para solucionar los problemas de desempleo o de precariedad del empleo, ya que, en realidad, se trata de ejercer las libertades que han sido reconocidas hace mucho tiempo en el plano formal (libertad de asociación y sindical, libertad de empresa, de trabajo, derecho a una vida familiar) y de las que están excluidas las personas que no pueden permitirse el lujo de perder su empleo y de vivir sin ingreso profesional (Supiot, 1999b). Promueve, por tanto, una nueva cultura del trabajo que va más allá del empleo, e incluye actividades sociales útiles y necesarias que están fuera de la esfera mercantil.

\subsection{Los mercados de trabajo transicionales}

El concepto de 'mercados de trabajo transicionales' desarrollado por Schmid trata de dar una alternativa, de manera que los mercados de trabajo flexibles puedan ser establecidos e incrementados sin dañar la necesidad de seguridad social y justicia (Schmid, 2002b: 151). Son una posibilidad de organizar la seguridad social como vía para incrementar la flexibilidad y la eficiencia económica. El punto de partida es que el crecimiento económico sostenido a través de la innovación es condición necesaria, pero no suficiente, para resolver la crisis del empleo. Incluso en los países que han tenido éxito para reducir el paro, para algunos grupos, la amenaza de desempleo de larga duración excluyente, o de flexibilidad en la forma de empleo precario de manera permanente, existe. El incremento de las desigualdades en la distribución 
de la renta y en las oportunidades de empleo son indicadores de la inapropiada organización del trabajo y del bienestar. La consecuencia de la nueva flexibilidad del mercado de trabajo no es sólo el desempleo, sino también la erosión de la cohesión social.

En los modernos mercados de trabajo, las transiciones entre la educación y el empleo remunerado se han vuelto más complicadas, incluso pueden empezar con desempleo, empleo parcial o trabajo no remunerado. Cuando las personas están empleadas a tiempo completo, pueden moverse entre varios estatus de empleo. Incluso cuando llega la edad de jubilación, puede que ciertos trabajadores vuelvan al mercado de trabajo. En definitiva, el ciclo de la vida es cada vez menos regular y cada vez hay más pasos sin orden entre varios estatus de empleo y no empleo (Schmid, 2002a).

La diferenciación y discontinuidad de las trayectorias de empleo son características fundamentales de los mercados de trabajo del futuro, y hacer frente a esto requiere la adquisición de unas habilidades que deben ser permanentemente renovadas y de una infraestructura con la que los individuos puedan contar si se requiere. Las transiciones del mercado de trabajo pueden ser vistas como acontecimientos críticos que conllevan tanto riesgos como oportunidades. Cada transición conlleva un riesgo de exclusión social, no sólo por el hecho de estar desempleado o desaventajado en el mercado de trabajo, sino también como consecuencia del desánimo que las personas pueden sufrir debido a las dificultades encontradas durante una transición crítica. El daño causado por tal proceso de exclusión estaría originado por los escasos roles alternativos que la sociedad ofrece. Los acuerdos institucionales por los que se establecen, regulan y promueven las transiciones son los mercados transicionales de trabajo. Los mercados de trabajo transicionales son foros para la negociación o el acuerdo de las relaciones de empleo variables. Contrastan con la idea del contrato de empleo estándar, que deja poco sitio para hacer ajustes a las circunstancias locales 0 a las necesidades individuales a lo largo de la vida. Crearían puentes fiables que facilitarían opciones adecuadamente institucionalizadas, calculables y socialmente legitimadas para negociar en las fases críticas (Schmid, 2002b: 180-188).

En tales circunstancias, se requiere un nuevo diseño del seguro de desempleo, o como mejor cabría denominarlo, seguro de empleo. Este seguro de empleo tendría tres pilares: el seguro de desempleo rediseñado, el seguro de movilidad y las políticas activas de mercado de trabajo. Estos tres pilares formarían un sistema coordinado de seguro de empleo que favorecería los mercados de trabajo transicionales. Se trata de acuerdos institucionales que empoderan a los individuos a transitar entre varios estatus de empleo durante su ciclo de vida, a través de un conjunto de opciones de movilidad y empleabilidad. Proporcionan seguridad de ingreso en las diferentes fases del ciclo de vida, y también en situaciones de desempleo, formación, discapacidad 0 retiro (Schmid, 2002a).

La propuesta de mercados de trabajo transicionales significaría una nueva cultura laboral en la que las fronteras entre actividad, inactividad, empleo, desempleo y formación se desdibujarían para satisfacer las necesidades y circunstancias de la persona en diferentes momentos de su vida.

\subsection{Flexiguridad en los mercados de trabajo}

Los dos conceptos anteriores tienen importantes conexiones con el concepto de flexiguridad, pero el uso de este término no se generaliza en el ámbito de las políticas de empleo hasta 2007, año en el que la Comisión Europea señala que los Estados miembros deberán integrar en sus políticas de mercado de trabajo los principios de flexibilidad y seguridad laboral. Desde el punto de vista teórico, la flexiguridad se plantea como la nueva receta contra el paro y los problemas de segmentación del mercado laboral. Una propuesta integral y equilibrada, en la que ambas partes de la relación laboral salen beneficiadas (win-win), donde se intercambian mayor flexibilidad en la gestión de la mano de obra por mayor seguridad de las personas trabajadoras.

Desde la perspectiva de su efecto en el mercado laboral, resulta crucial distinguir entre flexiguridad interna y externa. La interna propone flexibilidad en las condiciones laborales (salario, tiempo de trabajo, funciones) y seguridad (estabilidad) en el empleo. La externa aboga por la flexibilidad de entrada y salida en el empleo (catálogo de modalidades contractuales que se adapte a las necesidades empresariales y despido libre o, al menos, fácil), mientras que sitúa la seguridad no ya en el empleo, sino en el mercado de trabajo (políticas activas que llevarán a obtener un nuevo empleo con rapidez, y políticas pasivas que proporcionen seguridad económica en ese ínterin).

La Comisión Europea, en 2007, acoge el concepto oficial de flexiguridad, optando por la flexiguridad externa, en torno a tres elementos: flexibilidad de entrada y salida en el mercado de trabajo, políticas activas de empleo (información, orientación, y, sobre todo, formación profesional, en torno a la estrategia de aprendizaje permanente a lo largo de toda la vida) y políticas pasivas de empleo (que ofrezcan suficiencia protectora pero siempre condicionada a la búsqueda activa de empleo).

La aplicación de una propuesta de flexiguridad como la de la Comisión Europea ha generado aún más inseguridad en el caso de algunos países, puesto que ha reducido la protección al empleo en ausencia de un sistema potente de garantía de rentas y de políticas activas de mercado de trabajo. Un ejemplo es el caso de la economía española, cuya flexiguridad se ha acentuado a partir de la reforma laboral de $2012^{2}$.

${ }^{2}$ El sistema español es muy flexible, en la entrada y en la de salida. La contratación temporal es casi la norma en los nuevos contratos. 
En una línea muy diferente, hay diversas e interesantes aportaciones académicas sobre el concepto de flexiguridad y las medidas que se deberían adoptar para lograrla. Entre ellas, destacamos la que interpreta la flexiguridad como un nuevo pacto entre capital y trabajo (Wilthagen y Tros, 2004), la que incorpora la perspectiva del ciclo de vida (Klammer, 2004) y la que vincula la puesta en marcha de pactos de flexiguridad con la creación de mercados de trabajo transicionales. Nos parece que contienen elementos relevantes para ser tenidos en cuenta en una propuesta de flexiguridad que favorezca la competitividad, la creación de empleo y la inclusión social, bajo el respeto de los derechos sociales. Aportan elementos interesantes para una nueva cultura del trabajo en la que el diálogo entre los agentes sociales juega un papel fundamental.

\subsection{Las reducciones de la jornada laboral}

Desde mediados de los noventa, se proponen la reducción de la jornada laboral como medio para luchar contra el desempleo. Hubo acuerdos puntuales en grandes empresas al respecto, con el fin de hacer frente a los excesos de capacidad sin provocar despidos masivos. Sin embargo, solamente en el caso francés se estableció una normativa general para una jornada máxima de 35 horas semanales a partir del año $2000^{3}$. La legislación que acompañaba a la medida permitía a los empresarios imponer más horas por semana sin previo aviso y sin tener que pagarlas como horas extraordinarias, a condición de que el cómputo anual de horas no superara las 1.600. Además de la reducción del desempleo, la medida buscaba también equilibrar la vida laboral y la personal. El número de puestos de trabajo que se crearon como resultado de la reducción de la jornada resultó superior al previsto, dado que fueron años de importante expansión económica (De la Fuente y Zubiri, 2006: 88).

El concepto de la 'sociedad de media jornada' (Halbtagesgesellschaft) [Stahmer, 2006] señala que el desempleo no se reduce aferrándose a esperanzas de crecimiento económico, sino compartiendo el trabajo entre todos. Esta idea sugiere que el trabajo remunerado y el no remunerado deberían tener la misma importancia. Según los cálculos basados en una sociedad de media jornada, el volumen de trabajo remunerado de las mujeres se incrementaría, mientras que el de los hombres disminuiría con

\footnotetext{
El despido es libre y con indemnización (cada vez más reducida). Además, se trata de un sistema que no ofrece seguridad en el mercado de trabajo. Ello se manifiesta a través de la elevada tasa de paro que demuestra la insuficiencia de las políticas activas de empleo, cuyo gasto en porcentaje del PIB está a una distancia importante del que generaría seguridad en el mercado de trabajo. Las prestaciones por desempleo sólo son rentas sustitutivas durante un máximo de dos años. La reforma de $\mathbf{2 0 1 2}$ ha reducido la seguridad en el empleo sin que los mecanismos de seguridad en el mercado de trabajo se hayan reforzado.

${ }^{3}$ El contenido de esta normativa queda en parte vaciado a partir de 2008, dado que se modificó la ley para permitir a las empresas ampliar más las horas de trabajo.
}

referencia a como se estructura en la actualidad, llegando así a un equilibrio equitativo. El objetivo de la sociedad de media jornada es que tanto mujeres como hombres participen igualmente en el trabajo remunerado y no remunerado. El concepto del trabajo mezclado (Mischarbeit), según Brandl y Hildebrandt (2002), sigue ideales similares a los mencionados anteriormente. La concepción del trabajo contiene tanto la parte remunerada como la de reciprocidad.

De manera análoga, el concepto de 'nuevo trabajonueva cultura' (new work, new culture), formulado en los años ochenta por el filósofo Bergmann (2004), señala que el trabajo remunerado debería ascender sólo a aproximadamente 15-20 horas a la semana. Además, la gente debería estar capacitada para producir sus productos de consumo por sí misma (autoabastecerse) a través de centros de servicios descentralizados que ofrezcan información y ayuda tecnológica. Estos centros ayudarían también en la búsqueda y el encuentro de una actividad satisfactoria y útil para la persona. El objetivo del nuevo trabajo-nueva cultura no es la liberación del trabajo, sino su transformación para que, de esta manera, las personas sean individuos libres con autodeterminación 4 .

La crisis de 2008 reavivó los planteamientos en esta línea, pero integrados en propuestas más holísticas que incorporan tanto trabajo remunerado como no remunerado y, además, abordan la cuestión de la insostenibilidad ambiental del actual modelo de crecimiento. La New Economics Foundation (Coote, Franklin y Simms, 2010) propone una nueva norma de 21 horas de trabajo remunerado por semana, 0 su equivalente repartido en horas a lo largo del año. Sugiere esta cifra porque está muy cerca del tiempo medio que hombres y mujeres dedican al trabajo remunerado cada semana.

Este modelo nos llevaría a una buena vida más universal por dos razones. Primero, porque significaría una redistribución del trabajo remunerado más igualitaria entre hombres y mujeres y también entre personas desempleadas, ocupadas e inactivas. Segundo, porque si pasamos menos tiempo trabajando para cubrir nuestros hábitos de consumo, nos resultará más fácil hacer cosas que valoramos pero para las que ahora no tenemos tiempo: cuidar a los hijos e hijas y a otros familiares y amistades, pasar más tiempo con los demás, o

4 Un modelo de este concepto y su aplicación práctica se encuentra en la ciudad de Flint, cerca de Detroit, en los Estados Unidos. La decadencia de la industria automovilística en esta ciudad eminentemente dedicada a la producción en este sector fue muy grave. A partir del proyecto, los empleados trabajaban seis meses al año en la fábrica y los otros seis se dedicaban a una actividad auto-organizada que deseaban realizar. En este punto, estaban apoyados con conocimientos, análisis de potencial individual, formación y trabajo social, pero no con dinero. Por un lado, era importante encontrar los talentos, las capacidades, los valores y la ideología de los trabajadores; por otro, capacitarles para realizar sus ideas. Los trabajadores obtenían sólo la mitad de su salario, pero era suficiente. El proyecto ha sido exitoso. Los trabajadores cansados y resignados se han convertido en seres humanos con fuerza y perspectiva. Se ha creado una nueva cultura del trabajo en Flint. 
hacer trabajo voluntario, por ejemplo. Además, sería un modelo más sostenible medioambientalmente, puesto que implicaría que la gente ganaría menos y también consumiría menos, y la huella ecológica sería menor. Se trata de un planteamiento que encuentra buen acomodo en dos importantes corrientes de la economía no convencional: la economía feminista y la economía ecológica.

La propuesta considera los problemas transitorios que ocasionaría que nuestras economías convergiesen hacia una semana laboral de $\mathbf{2 1}$ horas. Entre ellos, destacan las resistencias de empresas, políticos/as y empleados/as, así como las dificultades económicas que ocasionaría a los trabajadores y trabajadoras que menos ganan en el mercado laboral. Como precondiciones para impulsar una medida de este tipo, se señalan una democracia fuerte y Gobiernos que rindan cuentas, algo que, desafortunadamente, no se cumple en buena parte del mundo, ni tan siquiera en los denominados países desarrollados o avanzados.

Hay que subrayar que las 21 horas no se plantean como receta, sino como provocación, cuestionando lo que se considera 'normal' y proponiendo pequeños pasos hacia una transformación radical, no como algo que pueda ser inmediatamente impuesto. Para alimentar el debate y estimular el pensamiento, se establecen sugerencias como:

- Alcanzar la reducción de horas laborables: ir reduciendo las horas gradualmente, mediante convenios en los que los salarios crezcan en una proporción menor que de otra manera no sería aceptable; reducir las horas extraordinarias; pasar de medidas de costo por empleado a medidas de costo por hora (en seguridad social).

- Garantizar un ingreso vital justo, aumentando el salario mínimo y las prestaciones, ofreciendo más y mejores servicios públicos, creando un comercio individual de emisiones de carbono, generando más actividad y consumo no mercantilizados.

- Mejorar las relaciones de género y la calidad de vida familiar mediante mayor flexibilidad en las relaciones de empleo; atención infantil universal de calidad; mayor cantidad de empleos en los cuidados y en la escuela primaria para los hombres, de manera que las actitudes sobre los trabajos masculinos y los femeninos vayan cambiando.

- Cambiar las normas y las expectativas mediante medidas como el apoyo gubernamental a las actividades no mercantilizadas y campañas que despierten la conciencia del valor del trabajo no remunerado.

Nos interesa resaltar no tanto la contribución de estas medidas a la creación de empleo, sino sobre todo el hecho de que en ellas el desempleo se aborda, no como problema individual de las personas que lo sufren, sino desde una perspectiva colectiva, que propone repartir las horas o los puestos de trabajo existentes entre un mayor número de trabajadores y trabajadoras. Un problema cuya solución pasa por transitar a una nueva cultura del trabajo.

\subsection{Renta básica universal y trabajo garantizado}

Las propuestas de renta básica universal no son recientes. Desde los noventa, hay líneas de investigación al respecto y desde 2002 existe una red internacional (Basic Income Earth Network). Se ha trabajado intensamente definiendo la propuesta, justificándola y también planteando su viabilidad presupuestaria en diferentes escenarios. A partir de

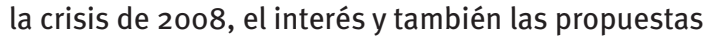
a favor de la renta básica universal se han reactivado.

La propuesta de un ingreso garantizado a toda la ciudadanía independientemente de su situación económica liberaría a las personas de tener que trabajar necesariamente para sobrevivir e impulsaría la mejora de la calidad de los peores empleos. De manera complementaria a la renta básica universal, en los últimos años, se ha definido la propuesta del trabajo garantizado. Se parte de la idea de que en nuestras sociedades existen muchos trabajos que no se hacen y ello significa que las necesidades no están cubiertas. Son trabajos de cuidado a personas dependientes, servicios de ocio y cultura, o de mantenimiento del entorno, entre otros. $\mathrm{El}$ Estado podría financiar y planificar estos trabajos, al tiempo que satisfaría las necesidades al actuar como garante del empleo para todas las personas. Se señalan algunas ventajas de esta propuesta frente a la de renta básica universal, como su carácter no inflacionista, su coordinación con el ciclo económico o la creación de servicios.

Las propuestas de expertos en el estudio de la desigualdad incluyen ambas medidas. Así, entre los quince pasos para acabar con la desigualdad propuestos por Anthony B. Atkinson (2016) figuran el de la creación de un ingreso básico o universal a escala nacional a todos los ciudadanos, aunque no trabajen y el de que el Estado se vuelva un empleador de última instancia, ofreciendo un empleo pagado al salario mínimo a todo aquel que lo solicite. Estas medidas precisan, como no podría ser de otra manera, una importantísima reforma hacia una fiscalidad progresiva, así como medidas para distribuir de manera más equitativa el patrimonio.

Estos planteamiento, sobre todo la renta básica universal, revolucionan el concepto de trabajo tal y como lo entendemos. El derecho a una seguridad económica mínima sin condiciones elimina la necesidad de trabajar para tener una vida digna y establece las condiciones para la libertad individual, para que cada persona haga lo que quiera con su vida. El trabajo garantizado, por su parte, responsabiliza a la toda la sociedad de buscar una actividad útil y remunerada a cada persona, y en definitiva, de la empleabilidad de trabajador o trabajadora. 


\subsection{El voluntariado empresarial y una nueva definición de pleno empleo}

El voluntariado empresarial ${ }^{5}$ tiene su origen en los Estados Unidos y su importancia ha crecido desde los años noventa. Se refiere a "cualquier apoyo formal organizado por la empresa en apoyo a los empleados y jubilados que deseen ofrecer su tiempo y habilidades de manera voluntaria en servicio de su comunidad" (Wild, 1993: 10). Debido al dinamismo del instrumento, la definición dada no comprende todas las formas de voluntariado empresarial en la actualidad. Por ello, se señalan una serie de características imperativas que definen el voluntariado empresarial (Wichelhaus, 2007): tiene que ser voluntario, es decir, el programa no es obligatorio para los trabajadores; debe constituir una aportación útil para la sociedad; es necesario que los voluntarios sean trabajadores o antiguos trabajadores de la empresa; y tiene que ser no remunerado, es decir, los trabajadores no pueden obtener una compensación financiera. En conclusión, el voluntariado empresarial tiene que ser un programa empresarial formal, y no sólo un apoyo bienintencionado de la dirección de la empresa (Halley, 1999; Peterson, 2006; Wild, 1993).

La razón primordial de la existencia del voluntariado empresarial es la de crear una imagen en la que las empresas forman parte de la comunidad y, por ello, interceden a favor de ésta. Lo que en estas páginas se plantea es que el voluntariado empresarial puede impulsar una nueva cultura del trabajo a través de algunos de los beneficios que genera para las empresas, los trabajadores, las organizaciones del tercer sector y que, posteriormente, se ven reflejados en los ciudadanos y toda la sociedad.

La gestión eficiente de la diversidad va a ser una de las tareas importantes en las organizaciones en el futuro. En el caso de las empresas, señalaremos que el desarrollo del voluntariado empresarial puede ayudar a adaptar una cultura empresarial y personal homogénea a la heterogeneidad en el ambiente de la empresa. La mano de obra está sensibilizada para la diversidad cultural y ética, y ello promueve la diversidad empresarial, la aceptación de diferencias y el desarrollo del sentido común (Schöffmann, 2001). Para los trabajadores, la participación en actividades de voluntariado empresarial, genera los siguientes beneficios vinculados con una nueva cultura del trabajo:

- Mejora su rol o posición en el mercado laboral, dado que puede suponer un aumento de la cualificación profesional y puede fomentar la autoconfianza de los trabajadores (Schöffmann, 2001).

- No menos importante es el hecho de que el voluntariado empresarial puede favorecer el

\footnotetext{
5 También denominado voluntariado corporativo (corporate volunteering).
}

equilibrio entre trabajo, tiempo libre y la familia (Backhaus-Maul, 2003; Schöffmann, 2001). En las actividades voluntarias, por ejemplo, compartir tiempo de ocio con personas con discapacidad, toda la familia, es decir, adultos y niños, puede involucrarse y así, aumentar el tiempo en actividades familiares. De esta manera, el tiempo en familia no resulta mermado por este tipo de compromisos sociales voluntarios en el tiempo libre.

- Las experiencias obtenidas de las actividades en un programa de voluntariado empresarial pueden ser un puente o una transición hacia un nuevo trabajo, una nueva actividad o una nueva fase de la vida de las personas (Schöffmann, 2001).

Por último, el voluntariado empresarial no sólo puede cambiar la vida laboral de los trabajadores ocupados, sino también de los desempleados. En este sentido, dado que la mano de obra existente tendrá periodos de voluntariado y no podrá estar disponible, la empresa tiene la posibilidad de emplear a más personas para contrarrestar la reducción del tiempo laboral de los voluntarios. La reducción del tiempo laboral estaría acompañada por la distribución de las actividades laborales y, de esta manera, sería posible disminuir el desempleo.

Para las organizaciones del tercer sector, el voluntariado empresarial incrementa los recursos de personal a su disposición y significa poder contar con profesionales con competencias y capacidades. Además, puede favorecer el acceso de estas organizaciones a redes sociales, a crear relaciones eficaces con los ciudadanos, con la sociedad y con las empresas, lo cual puede facilitar el acceso a financiación. Por otro lado, la actividad voluntaria en el tercer sector facilita la participación, la colaboración y la cooperación social a través del trabajo no remunerado, lo cual favorece la reproducción de valores comunes y solidarios.

El voluntariado empresarial podría apoyar una nueva definición de pleno empleo a través de una distribución del trabajo, la disminución de la jornada laboral, y de las transiciones difusas entre trabajo remunerado y otras actividades útiles. La reducción de la jornada laboral por el tiempo dedicado a las actividades voluntarias establecería nuevas posibilidades de empleo para personas desempleadas. Las empresas tendrían la posibilidad contratar y emplear más trabajadores, y distribuirían el trabajo entre más personas en el mercado laboral. Sin embargo, hay que tener en cuenta que las actividades del voluntariado empresarial están restringidas a actividades en el tercer sector, así que queda pendiente de definir la cuestión de cómo se integran las actividades útiles del entorno familiar o de reciprocidad (trabajo doméstico y cuidado de personas dependientes). Para que el voluntariado empresarial tuviese además efectos positivos en la igualdad de género, estas actividades de reciprocidad deberían estar integradas como posibles formas de desarrollo del voluntariado empresarial. 
Por otro lado, las actividades del voluntariado empresarial otorgan un nuevo valor a diferentes formas de trabajo dentro de la sociedad. Intensifican la participación social y cívica de los trabajadores e implican, de esta manera, nuevos impulsos sociales y solidarios, más sensibilidad en cuanto al rol del trabajo remunerado y no remunerado de cada individuo, y hacia nuevas ideas sostenibles para el diseño de las transiciones entre las diferentes etapas de la vida del trabajador.

\subsection{La inversión en infraestructura social como generadora de empleo: una propuesta feminista}

A raíz de la crisis de 2008 , han surgido numerosos trabajos que ilustran los efectos de las políticas de austeridad implementadas. Algunos de ellos señalan que las medidas de austeridad no están justamente distribuidas entre la población, y que los hogares con menor nivel de ingresos y las personas de más edad resultan más afectados (Ginn, 2013).

Los trabajos de las corrientes de la economía feminista han puesto de manifiesto el hecho de que las políticas de austeridad tienen un claro efecto de género. Las mujeres y los hombres tienen diferentes comportamientos ante los cambios en las políticas, debido a que ocupan diferentes posiciones sociales y a que se ven afectados de distinta manera por las normas sociales. Se señalan tres grandes roles del Estado social cuya contracción es especialmente significativa para las mujeres y para el progreso de la igualdad de género (Rubery, 2015):

- El primero se refiere al papel del Estado como fuente de renta y señala que los efectos inmediatos de los recortes en subsidios por hijos e hijas, desempleo o vejez en el conjunto la población se intensifican en el caso de las mujeres. La razón no es otra que el modelo de empleo femenino existente, con más parcialidad, menor remuneración o interrupciones en la carrera profesional. Ello implica una mayor dependencia de las mujeres de las prestaciones y subsidios, lo cual desencadena una mayor vulnerabilidad entre ellas ante los recortes en el gasto social.

- El segundo es el relativo a los servicios públicos que soportan la reproducción social, cuya reducción puede implicar una intensificación del trabajo femenino, fundamentalmente del no remunerado. El incremento del trabajo no remunerado en los hogares, combinado con el desigual reparto de las tareas domésticas y de cuidado entre mujeres y hombres, provoca que un buen número de mujeres se retiren del mercado laboral durante las crisis (Gálvez y Rodríguez, 2013a).

- Y el tercero hace referencia al papel del sector público como empleador. Los recortes en servicios públicos como la educación, la salud o los servicios sociales afectan a las mujeres también como empleadas en ellos, dado que un elevado porcentaje del empleo femenino está en el sector público. Recientes análisis para el caso de cinco países europeos ${ }^{6}$ muestran que, a consecuencia de la mayor concentración de las mujeres trabajadoras en el sector público, es probable que las medidas de austeridad tengan un efecto desproporcionado en el empleo femenino (Grimshaw, Rubery y Marino, 2012).

Estos enfoques sobre la intensificación de los efectos adversos de la crisis entre las mujeres se han ocupado, asimismo, de hacer propuestas para evitar que ellas no se queden al margen de la recuperación económica. Las recientes aportaciones en esta línea toman como punto de partida la idea de que es necesario adoptar un marco macroeconómico expansivo liderado por la inversión pública. Se trataría de un enfoque para la recuperación económica que combine una mejor financiación de los sistemas de salud y de protección social, y su coordinación con políticas impositivas y de empleo. Lo han denominado feminist economic strategy 0 Plan F (Bargawi, Cozzi y Himmelweit, 2017), o purple economy (Ilkkaracan, 2017).

La principal base de estos enfoques pasa por que los Gobiernos amplíen lo que se entiende por economía, de manera que se incluya la producción de bienes y servicios llevada a cabo mediante trabajo no remunerado. Dicha ampliación se refiere, particularmente, al trabajo de cuidados, del que se siguen ocupando fundamentalmente las mujeres, a pesar de su creciente incorporación al trabajo mercantil.

Se trataría de revertir los recortes de los últimos años y de proporcionar servicios públicos que reduzcan el trabajo no remunerado, tanto el doméstico como el de cuidados. No se trataría de abolir (mercantilizar) todo este trabajo, sino de repartirlo de manera equitativa entre mujeres y hombres. Para ello, es necesario modificar las regulaciones de empleo y de protección social, por ejemplo, incrementando el tiempo de permiso parental reservado a padres, eliminando las penalizaciones que supone el trabajo a tiempo parcial o fomentando una semana laboral de 30 horas tanto para hombres como para mujeres (Elson, 2017: 18).

Otro aspecto importante a considerar es la financiación de estos servicios relativos a la salud, la educación y los cuidados. Normalmente el gasto en ellos es clasificado en el presupuesto público como gasto corriente, lo cual impide a los Gobiernos endeudarse para su financiación. Ello no ocurre con partidas dedicadas a las infraestructuras físicas (carreteras, ferrocarriles), ni siquiera con el gasto militar. Son consideradas inversiones que en el futuro van a beneficiar a las generaciones venideras $y$, por ello, se considera adecuado el endeudamiento

${ }^{6}$ Entre el $67 \%$ y el $74 \%$ para el caso de Suecia, Reino Unido, Alemania, Hungría y Francia. 
para su financiación. Pues bien, lo que las corrientes de la economía feminista consideran es que los recursos dedicados a la ampliación de los servicios de salud, de cuidados y educativos deben ser también considerados como inversión. En este caso, se trata de inversión en infraestructura social, dado que estos servicios expanden la producción futura y el bienestar humano, creando y manteniendo el denominado 'capital humano'. Ello genera empleos remunerados decentes tanto para las mujeres como para los hombres, reduce el trabajo no pagado realizado por las mujeres y aumenta la producción y el bienestar futuros (Elson, 2017: 19).

La 'economía púrpura' (purple economy) es una alternativa para un orden económico sostenible e igualitario desde el punto de vista del género. Se organiza en torno a la sostenibilidad del trabajo de cuidados, a través de la internalización de los costes del cuidado en el sistema. Ello implica la provisión pública universal de servicios de cuidado para niños y niñas, y personas mayores, enfermas o discapacitadas. Dado que se reconoce la dependencia del trabajo de cuidados como un componente indispensable del bienestar y se reordenan las prioridades situando la crianza (mantenimiento) de los seres humanos en el centro (Ilkkaracan, 2017: 29), podemos decir que esta perspectiva avanza decididamente hacia una nueva cultura del trabajo.

\section{Reflexiones finales}

No cabe duda de que el problema del desempleo tiene raíces económicas y que parte de las medidas a emprender para luchar contra él deben considerar aspectos relacionados con la actividad de los sectores económicos, el ajuste entre las características de la mano de obra y los puestos de trabajo generados en esos sectores, y también los mecanismos de fijación de las rentas del trabajo y del capital. Sin embargo, desde nuestro punto de vista, se adopta un esquema fragmentario y reduccionista, que ignora que el problema del desempleo está también relacionado con cuestiones que van más allá de lo económico y que son de índole sociopolítica, organizativa y cultural.
Desde una perspectiva holística, además de considerarse los condicionantes económicos, se hace también referencia al modelo sociolaboral, a los diferentes tipos de trabajos, tareas y roles asignados a las personas que componen la sociedad, que vienen condicionados, entre otras cosas, por la organización de los centros de trabajo, el reparto de las tareas en los hogares, la protección social existente para situaciones de no empleo, los valores sociales predominantes, entre otras cuestiones.

Se trataría de avanzar hacia una nueva cultura del trabajo en la que las tareas no remuneradas desarrolladas en los hogares, el tiempo dedicado a la formación, las actividades sindicales, políticas, artísticas y culturales, así como las de voluntariado llevadas a cabo en las organizaciones del tercer sector serían económica y socialmente reconocidas y valoradas. Como ventajas adicionales, cabe señalar, en primer lugar, que se abordaría la denominada 'crisis de los cuidados', el sobreesfuerzo de la doble jornada y la 'doble presencia' que principalmente soportan las mujeres. En segundo lugar, y no menos importante, los posibles empleos que se generarían en servicios relacionados con la salud, los cuidados y la educación no sufrirían la constante amenaza de deslocalización hacia regiones o países con bajos costes y legislaciones laborales más laxas.

Lo que aquí se ha presentado es un breve recorrido por algunas de las propuestas para hacer frente a la escasez de puestos de trabajo y a la inestabilidad laboral de las últimas décadas. Con toda seguridad, su implementación precisa del acuerdo de las instituciones sociolaborales y exige, por tanto, un importantísimo esfuerzo de diálogo, lo que requiere un prolongado periodo de adaptación. Ello no significa que no contengan parte de las soluciones a uno de los mayores retos a los que se enfrentan las actuales sociedades: proporcionar un lugar 0 actividad socialmente legitimada y que permita vivir dignamente a toda la población. En definitiva, se trataría de avanzar hacia mercados de trabajo y sociedades más inclusivas y, probablemente, más cohesionadas y equitativas. 
ATKINSON, A. B. (2016): Desigualdad: ¿qué podemos hacer?, Fondo de Cultura Económica.

BACKHAUS-MAUL, H. (2003): “Engagementförderung durch unternehmen in den USA: Über die produktive balance zwischen erwerbsarbeit, familienleben und bürgerschaftlichem engagement. Apoyo del compromiso social por empresas en los EE.UU.: sobre el balance productivo entre trabajo remunerado, vida familiar y compromiso cívico, en COMISIÓN PARLAMENTARIA DEL BUNDESTAG (ed.), Bericht bürgerschafltliches engagement: Auf dem weg in eine zukunftsfähige bürgergesellschaft [Reporte compromiso cívico: el camino hacia una sociedad cívica del futuro], págs. 85-148. Opladen: Leske+Budrich.

BARGAWI, H.; COZZI, G.; y HIMMELWEIT, S. (2017): Economics and Austerity in Europe: Gendered Impacts and Sustainable Alternatives, Routledge.

BERGMANN, F. (2004): Neue Arbeit, neue Kultur [Nuevo trabajo, nueva cultura], Freiamt, Arbor.

BRANDL, S.; y HILDEBRANDT, E. (2002): Zukunft der Arbeit und soziale Nachhaltigkeit: zur Transformation der Arbeitsgesellschaft vor dem Hintergrund der Nachhaligkeitsdebatte [Futuro del trabajo y sostenibilidad social. Hacia la transformación de la sociedad del trabajo con el debate de sostenibilidad de fondo], Opladen, VS Verlag für Sozialwissenschaften.

CASTEL, R. (1997): Las metamorfosis de la cuestión social. Una crónica del salariado, Barcelona, Paidós.

COMISIÓN EUROPEA (2007): Comunicación de la Comisión al Parlamento Europeo, al Consejo, al Comité Económico y Social Europeo, y al Comité de las Regiones, de 27 de junio de 2007. Hacia los principios comunes de la flexiguridad: más y mejor empleo mediante la flexibilidad y la seguridad, Bruselas, Comisión Europea, COM (2007) 359 final.

COOTE, A.; FRANKLIN, J.; y SIMMS, A. (2010): 21 Horas: por qué una semana laboral más corta puede ayudarnos a prosperar en el siglo XXI, New Economics Foundation.

DE LA FUENTE, M.; y ZUBIRI, J. B. (2006): “Efectos sobre el empleo de la reducción del tiempo de trabajo: aprendizajes de la experiencia francesa", Lan Harremanak, nํㅜ 34, págs. 79-105.

ELSON, D. (2017): “A gender-equitable macroeconomic framework for Europe", en BARGAWI, H.; COZZI, G.; y HIMMELWEIT, S., Economics and Austerity in Europe: Gendered Impacts and Sustainable Alternatives, Routledge.

GÁLVEZ, L.; y RODRÍGUEZ P. (2013a): La desigualdad de género en las crisis económicas, serie Materiales CIP, nำ 7, Institut de Ciènces Polítiques i Socials.

- (2013b): "El empleo de las mujeres en la España democrática y el impacto de la Gran Recesión", Revista Internacional de Ciencias Sociales, nํㅜ 32, págs. 105-123.

GINN, J. (2013): "Austerity and inequality. Exploring the impact of cuts in the UK by gender and age", Hipatia Press, vol. 1, nํㅜ 1, págs. 28-53.

GRIMSHAW, D.; RUBERY, J.; y MARINO, S. (2012): “Public sector pay and procurement in Europe during the crisis: The challenges facing local government and the prospects for segmentation, inequalities and social dialogue" [proyecto de la Comisión Europea, VS/2011/0141]. 
HALLEY, D. (1999): Employee Community Involvement Gemeinnütziges Arbeitnehmerengagement. Ein vollständiger Leitfaden für Arbeitgeber, Arbeitnehmer und gemeinnützige Organisationen [Employee Community Involvement - Compromiso de trabajador de utilidad pública. Guía completa para empresarios, trabajadores y organizaciones de utilidad pública], Colonia, Fundus.

ILKKARACAN, I. (2017): “A feminist alternative to austerity: The purple economy as a gender-egalitarian strategy for employment generation", en BARGAWI, H.; COZZI, G.; y HIMMELWEIT, S., Economics and Austerity in Europe: Gendered Impacts and Sustainable Alternatives, Routledge.

KLAMMER, U. (2004): "Flexicurity in a life-course perspective", European Review of Labour and Research, vol. 10, nํ2, págs. 282-299.

PAUGAM, S. (2000): Le salarié de la précarité, París, PUF.

- (1996b): “La construction d'un paradigme”, en PAUGAM, S. (dir.), L'exclusion, l'état des saviors, París, La Découverte.

PETERSON, D. K. (2006): "Recruitment strateges for encouraging participation in corporate volunteer programs", Journal of Business Ethics, vol. 49, nํㅜ 4, págs. 371-386.

RUBERY, J. (2015): "Austerity, the public sector and the threat to gender equality", The Economic and Social Review, vol. 46, no - 1, págs. 1-27.

SCHMID, G. (2002a): "Employment insurance in critical transitions during the life-course", simposio 'The Future of Work, Employment and Social Protection: The Dynamics of Change and the Protection of Workers', Lyon, $17 / 18$ enero.
- (2002b): "Towards a theory of transitional labour markets”, en SCHMID, G.; y GAZIER, B. (eds.), The Dynamics of Full Employment. Social Integration through Transitional Labour Markets, Edward Elgar.

SCHÖFFMANN, D. (2001): "Vom Nutzen des corporate Volunteering" [Sobre la utilidad del voluntariado corporativo], en SCHÖFFMANN, D., (ed.), Wenn alle gewinnen. Bürgerschaftliches Engagement von Unternehmen [Cuando todos ganan. Compromiso cívico de las empresas], Hamburgo, Körber-Stiftung, págs. 95-104.

STAHMER, C. (2006): Halbtagesgesellschaft. Anregungen für ein sozial nachhaltiges Deutschland [Sociedad de media jornada. inspiración para una Alemania social sostenible], Bielefeld.

SUPIOT, A. (1999a): Trabajo y empleo. Transformaciones del trabajo y futuro del Derecho del trabajo. Informe para la Comisión Europea, Valencia, Tirant lo Blanch.

- (1999b): “Travail, droit et lien social” [conferencia], Ginebra, Organización Internacional del Trabajo, Instituto Internacional de Estudios Sociales [rhttp://www.infotheque.info/ cache/8833/www-ilo-mirror.cornell.edu/ public/french/bureau/inst/papers/publecs/ supiot/index.htm〉].

WICHELHAUS, P. (2007): Corporate Volunteering, Saarbrücken, VDM Verlag Dr. Müller e. K.

WILD, C. (1993): Corporate Volunteering Programs: Benefits to Business, Nueva York, Conference Board.

WILTHAGEN, T.; y TROS, F. (2004): “The concept of flexicurity: A new approach to regulating employment and labour markets", European Review of Labour and Research, nํㅡㄹ 2, págs. 166-186. 